\title{
Finding zeros of the Riemann zeta function by periodic driving of cold atoms
}

\author{
C. E. Creffield \\ Departamento de Física de Materiales, Universidad Complutense de Madrid, E-28040, Madrid, Spain
}

G. Sierra

Instituto de Física Teórica, UAM-CSIC, E-28049, Madrid, Spain

(Received 11 November 2014; published 8 June 2015)

\begin{abstract}
The Riemann hypothesis, which states that the nontrivial zeros of the Riemann zeta function all lie on a certain line in the complex plane, is one of the most important unresolved problems in mathematics. We propose here an approach to finding a physical system to study the Riemann zeros, which is based on applying a time-periodic driving field. This driving allows us to tune the quasienergies of the system (the analog of the eigenenergies for static systems), so that they are directly governed by the zeta function. We further show by numerical simulations that this allows the Riemann zeros to be measured in currently accessible cold-atom experiments.
\end{abstract}

DOI: 10.1103/PhysRevA.91.063608

PACS number(s): 03.75.Lm, 02.10.De, 02.30.Gp

\section{INTRODUCTION}

The Riemann hypothesis states that the nontrivial zeros of the Riemann function, $\zeta(s)$, have the form $s_{n}=1 / 2 \pm i E_{n}$, where the $E_{n}$ are all real. A fascinating approach to treating the Riemann problem is to consider the $E_{n}$ to be eigenvalues of a self-adjoint operator, since if such an operator could be identified the $E_{n}$ would necessarily be real. This idea, known as the Pólya and Hilbert conjecture, is supported by much evidence (see Refs. [1,2] for reviews), notably that the zeros appear to closely follow the Gaussian unitary ensemble (GUE) statistics [3-6] of random matrix theory and quantum chaos. This unexpected connection between number theory and physics has inspired many suggestions for finding physical implementations of the Riemann function, such as evaluating the Fourier transform of a suitable wave using optomechanical means [7] or far-field diffraction [8], or by measuring entanglement in quantum systems [9].

In this work we propose a very different approach, in which a time-periodic driving potential is used to modify the dynamics of a quantum system. Because the system is periodically driven its dynamics is not described by energy eigenvalues but by a generalization of these quantities termed "quasienergies." Our central result is the construction of a driving field for which the corresponding quasienergy spectrum is given by the Riemann $\Xi$ function $[10,11]$, or by a smoothed version of this function, $\Xi^{*}$, introduced by Pólya. This last has the appealing feature that the appropriate driving field can be expressed in a simple closed form. In both cases the zeros of the $\Xi$ function correspond to degeneracies, or crossings, of the quasienergies. These are of particular physical significance because they correspond to the phenomenon known as "coherent destruction of tunneling" (CDT) in which the dynamics of the system is frozen [12]. As an example we show how the zeros can be seen directly in cold-atom experiments by measuring the expansion rate of a condensate held in a driven optical lattice. This technique thus represents a different and powerful way of finding a physical realization of the Riemann function.

\section{METHOD}

We begin by considering a standard two-level system, driven by a time-periodic function $f(t)=$
$f(t+T)$ :

$$
H(t)=-J \sigma_{x}+f(t) / 2 \sigma_{z},
$$

where $J$ is the tunneling between the two levels. As $f(t)$ is time periodic, the natural framework to treat the problem is given by Floquet theory [13]. In this approach one seeks the eigensystem of the Floquet operator

$$
\mathcal{H}(t)=H(t)-i \partial_{t},
$$

where we have set $\hbar=1$. Henceforth we shall also measure all energies (and frequencies) in units of $J$. The eigenstates of $\mathcal{H}(t)$ are $T$-periodic functions called Floquet states, and their associated eigenvalues, which play an analogous role to energy eigenvalues for the case of a static Hamiltonian, are called quasienergies. The Floquet states provide a complete basis, and expanding the wave function in these states provides similar advantages to the normal procedure of expressing a state in energy eigenstates in the undriven case.

In general it is difficult to obtain analytical expressions for the Floquet states. In the strong-driving limit, however, when the frequency $\omega=2 \pi / T$ is the dominant energy scale, it is possible to make an expansion by first solving just for the time-dependent component of $H(t)$ and applying the static part as perturbation [14]. In this way one obtains a perturbative series in orders of $J$. Truncating at first-order gives the simple result $H_{\text {eff }}=-J_{\text {eff }} \sigma_{x}$, where the effective tunneling $J_{\text {eff }}$ is given by

$$
J_{\text {eff }} / J=\frac{1}{T} \int_{0}^{T} d t e^{-i F(t)},
$$

and $F(t)=\int_{0}^{t} d t^{\prime} f\left(t^{\prime}\right)$. The quasienergies are given simply by the eigenvalues of $H_{\text {eff }}$, namely $\epsilon_{ \pm}= \pm\left|J_{\text {eff }}\right|$.

From Eq. (3) it is thus straightforward to calculate the behavior of the quasienergies (or equivalently, of the effective tunneling) for a given driving potential $f(t)$. For the case $f(t)=K \cos \omega t$, for example, this yields the wellknown Bessel function renormalization [15] of tunneling $J_{\text {eff }}=J \mathcal{J}_{0}(K / \omega)$. At zeros of the Bessel function, $K / \omega=$ $2.404,5.520, \ldots$, the effective tunneling vanishes, producing CDT. This effect has been measured experimentally [16-18] in the dynamics of driven ultracold atoms. 
We need, however, to solve the inverse problem; to find an $f(t)$ that produces a given behavior of the quasienergies. We shall first explain our technique using Pólya's function, $\Xi^{*}(E)$, because this gives a convenient closed form for the solution, and then go on to consider the more complicated case of the true Riemann $\Xi$ function.

\section{A. Driving function for Pólya's function}

Pólya's function is given by [19]

$$
\Xi^{*}(E)=4 \pi^{2}\left[K_{a+i E / 2}(x)+K_{a-i E / 2}(x)\right],
$$

where $K_{\beta}(t)$ is the modified $K$-Bessel function, $x=2 \pi$, and $a=9 / 4$. This is a smoothed version of the Riemann $\Xi$ function

$$
\Xi(E)=\frac{1}{2} s(s-1) \Gamma\left(\frac{s}{2}\right) \pi^{-s / 2} \zeta(s), \quad s=1 / 2+i E,
$$

whose zeros coincide with the nontrivial zeros of $\zeta(s)$. Although, as Titchmarsh pointed out [20], Pólya's zeta function cannot truly be regarded as an approximation to $\Xi(E)$ in the most obvious sense, they do share many properties. Most importantly, $\Xi^{*}(E)$ has the same average distribution of zeros $[11,20]$, following the smooth term of the RiemannMangoldt formula, and so nonetheless represents an interesting application of our method. Pólya further proved that the zeros of $\Xi^{*}(E)$ are real for any value of the constant $a$. The spectrum of the $x p$-type [21] and Dirac Hamiltonian [22] is given, for example, by the zeros of Eq. (4) with $a=1 / 2$.

The modified Bessel function can be conveniently expressed as the integral identity

$$
K_{\beta}(x)=\int_{0}^{\infty} d t \cosh (\beta t) e^{-x \cosh t},
$$

and thus

$$
\begin{aligned}
\Xi^{*}(E) & =8 \pi^{2} \operatorname{Re}\left[K_{a+i E / 2}(2 \pi)\right] \\
& =8 \pi^{2} \int_{0}^{\infty} d t \cosh (a t) e^{-2 \pi \cosh t} \cos (E t / 2) .
\end{aligned}
$$

We are aiming to obtain the result $\operatorname{Re}\left[J_{\text {eff }}(E)\right] \propto \Xi^{*}(E)$. Combining Eqs. (3) and (7) reveals that this requires $F(t)$ to obey the relation

$$
\int_{0}^{T} d t \cos F(t)=\int_{0}^{\infty} d t \alpha \cosh (a t) e^{-2 \pi \cosh t} \cos (E t / 2),
$$

where $\alpha$ is an arbitrary constant. Because the integrand on the right-hand side decays rapidly with $t$, we can replace the upper limit of integration with $T$, as long as we take $T$ to be sufficiently large. In this case we can then write [23]

$$
F(t)=\cos ^{-1}\left[\alpha \cosh (a t) e^{-2 \pi \cosh t} \cos (E t / 2)\right] .
$$

The value of $\alpha$ is now fixed by noting that we require $F(0)=0$, and thus $\alpha=e^{2 \pi}$. The driving field is then given by $f(t)=$ $\partial_{t} F(t)$, yielding the final result

$$
f(t)=-\frac{\phi(t)[a \tanh a t-2 \pi \sinh t-(E / 2) \tan (E t / 2)]}{\sqrt{1-\phi(t)^{2}}},
$$

where $\phi(t)=\cosh (a t) e^{2 \pi(1-\cosh t)} \cos (E t / 2)$.

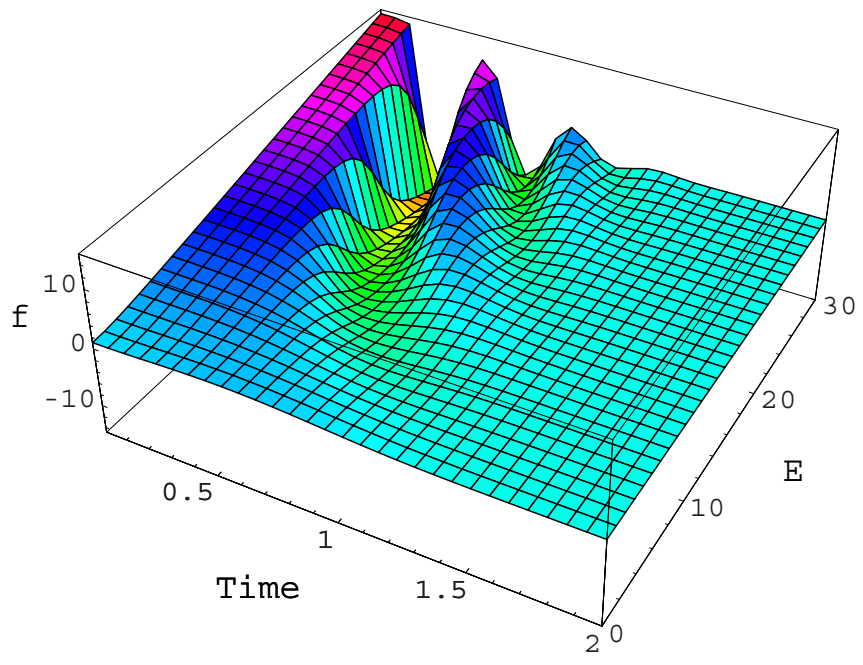

FIG. 1. (Color online) Surface plot of the time-dependent driving function $f(t)$ for the smoothed function $\Xi^{*}(E)$ [Eq. (10)]. Energy is measured in units of the tunneling $J$, and time in units of $J^{-1}$. For small values of $E$ the function is quite featureless, but progressively increases in amplitude and develops more oscillations as $E$ increases. The corresponding driving function for $\Xi(E)$ behaves similarly (see Fig. 4).

In Fig. 1 we show the form of $f(t)$ as $E$ is varied. Little structure is visible for $t>1.5$. The reason is the rapid decrease of $\phi(t)$ with $t$, arising from the exponential term $e^{-2 \pi \cosh t}$. Indeed, $|\phi(t)|<1.3 \times 10^{-6}, \forall E$ and $t>2$. Accordingly we now set the period of the driving, and thus the cutoff in the integration in Eq. (8), to be $T=\pi / 2$. We shall use this value throughout the rest of the paper. In Fig. 2(a) we show the full periodic driving field, obtained by periodically repeating cycles of $f(t)$. Although this form of $f(t)$ indeed satisfies Eq. (8), the driving can be made more effective by imposing a further set of conditions on it: $(i)$ to avoid heating in the coldatom model (see Sec. III C), the average of $f(t)$ over one period should vanish, (ii) discontinuities should be avoided, and (iii) for the quasienergy crossings to be well defined, the Floquet states must be from different parity classes. If condition (iii) is not fulfilled, the von Neumann-Wigner theorem implies that the quasienergies cannot cross as $E$ is varied and will instead form a broad avoided crossing. As the system is periodically driven, the appropriate generalized parity operator involves both inversion and time translation $\mathcal{P}: x \rightarrow-x, t \rightarrow$ $t+T / 2$. These three conditions can be satisfied by joining four copies of the fundamental waveform (10) as shown in Fig. 2(b), to create what amounts to two pulses of opposite sign, with a total period of $T_{0}=4 T$.

\section{B. Driving function for $\Xi(E)$}

We now turn to the more important case of the Riemann function itself. This can be written as

$$
\Xi(E)=\int_{0}^{\infty} d t \Phi(t) \cos (E t / 2)
$$

where $\Phi(t)=2 \pi e^{5 t / 4} \sum_{n=1}^{\infty}\left(2 \pi e^{t} n^{2}-3\right) n^{2} e^{-\pi n^{2} e^{t}}$, as defined in Ref. [10]. We now follow the same procedure as before, 

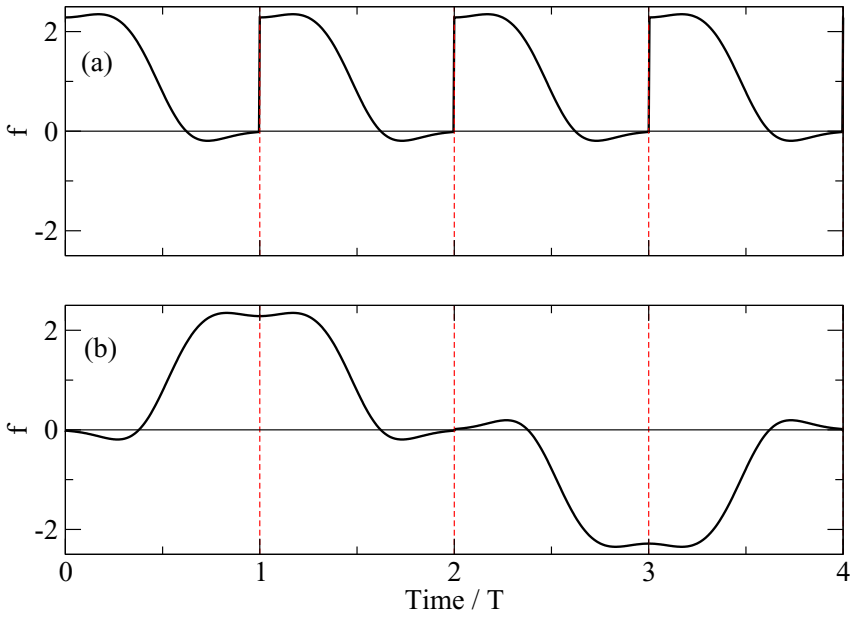

FIG. 2. (Color online) (a) The driving potential $f(t)$ is extended to be a periodic function by periodically repeating its behavior over the interval $0 \leqslant t<T$. This produces, however, a function with rather poor performance. (b) Construction of a more efficient driving potential. Four copies of $f(t)$ are joined together, following some reflection transformations, to create a single continuous function, resembling two localized pulses of opposite sign. The vertical dashed lines indicate where the segments are joined. This function is continuous, has a time-average of zero, and $f(t)=-f\left(t+T_{0} / 2\right)$ where $T_{0}=4 T$ is the total period of the signal, satisfying the parity requirement given in the text.

seeking a driving potential such that $\operatorname{Re}\left[J_{\text {eff }}(E)\right] \propto \Xi(E)$. This requires finding the solution of the equation

$$
\int_{0}^{T} d t \cos F(t)=\alpha \int_{0}^{\infty} d t \Phi(t) \cos (E t / 2),
$$

where $\alpha$ is a constant. We show the behavior of $\Phi(t)$ in Fig. 3 . Since $\Phi(t)$ decreases rapidly with $t$, being fitted reasonably well by a simple Gaussian function [8], we can substitute the upper limit of integration on the right-hand side of Eq. (12) to be $T$, as long as $T$ is sufficiently large for the integral to converge. As before, we take $T=\pi / 2$. We can then write

$$
F(t)=\cos ^{-1}[\alpha \Phi(t) \cos (E t / 2)]
$$

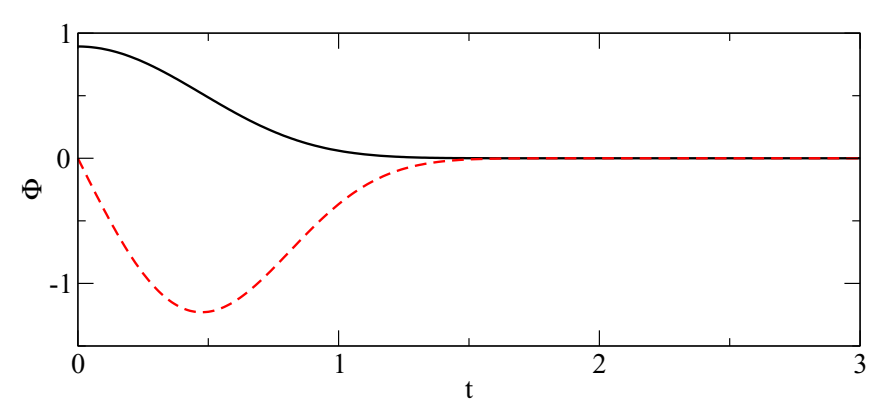

FIG. 3. (Color online) Behavior of $\Phi(t)$ and its derivative. Solid black line: $\Phi(t)$ has a global maximum at $t=0$, for which $\Phi(0) \simeq$ 0.89339 and decays rapidly as $t$ increases. Dashed red line: the derivative, $\Phi^{\prime}(t)=\partial_{t} \Phi(t)$, evaluated from Eq. (15). The series expansions for both $\Phi(t)$ and $\Phi^{\prime}(t)$ were truncated at 30 terms. Time is measured in units of $J^{-1}$.
Imposing the boundary condition $F(0)=0$ requires $\alpha$ to take the value $\alpha=1 / \Phi(0)$. As $\Phi(0)$ is the global maximum of the function, this guarantees that $|\alpha \Phi(t)| \leqslant 1$ for all values of $t$, and thus $F(t)$ is real and well defined.

Having obtained $F(t)$ it is now straightforward to calculate $f(t)=\partial_{t} F(t)$. This yields the final result

$$
f(t)=-\frac{\left[\Phi^{\prime}(t) \cos (E t / 2)-(E / 2) \Phi(t) \sin (E t / 2)\right]}{\sqrt{\Phi^{2}(0)-[\Phi(t) \cos (E t / 2)]^{2}}} .
$$

The derivative of $\Phi(t)$ can be evaluated by differentiating its series expansion term by term, to give

$$
\Phi^{\prime}(t)=\frac{\pi e^{5 t / 4}}{2} \sum_{n=1}^{\infty}\left(30 e^{t} n^{2} \pi-8 e^{2 t} n^{4} \pi^{2}-15\right) n^{2} e^{-\pi n^{2} e^{t}}
$$

which we also show in Fig. 3.

We show the full behavior of $f(t)$ as a function of time and the parameter $E$ in Fig. 4. Despite the complicated form of Eq. (14), this plot shows a striking similarity to Fig. 1, in which the $f(t)$ giving rise to $\Xi^{*}(E)$ is shown. This should not be unexpected because $\Xi^{*}(E)$ is simply a smoothed version of the Riemann function, and consequently the main features of the driving functions must be the same.

Just as for the case of the smoothed Riemann function, a more effective driving potential is obtained by joining four copies of $f(t)$ to produce a continuous function with a definite parity and zero time average. In Fig. 5 we show the form of the driving function we obtain in this way and compare it with the driving function that produces $\Xi^{*}(E)$. Examining the two curves in detail reveals that, although the functions share the same general form (as seen in the similarity of the two surface plots Figs. 1 and 4), there are nonetheless significant small differences. These minor differences lead to the different

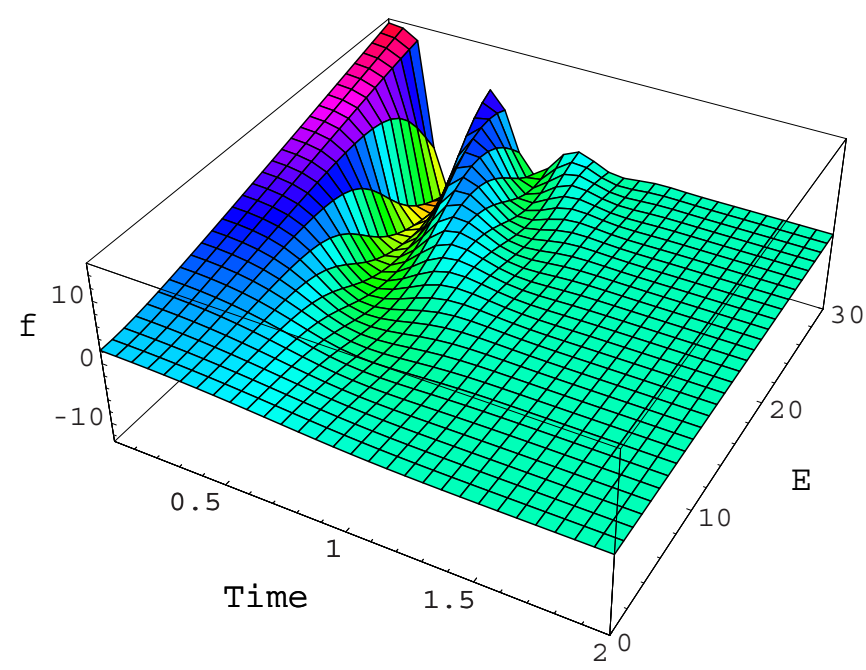

FIG. 4. (Color online) Surface of the driving function $f(t)$ [Eq. (14)], which produces a quasienergy spectrum proportional to the Riemann $\Xi$ function. The behavior resembles strongly the driving function for the smoothed Riemann function, shown in Fig. 1. As before, energy is measured in units of $J$, and time in units of $J^{-1}$. 


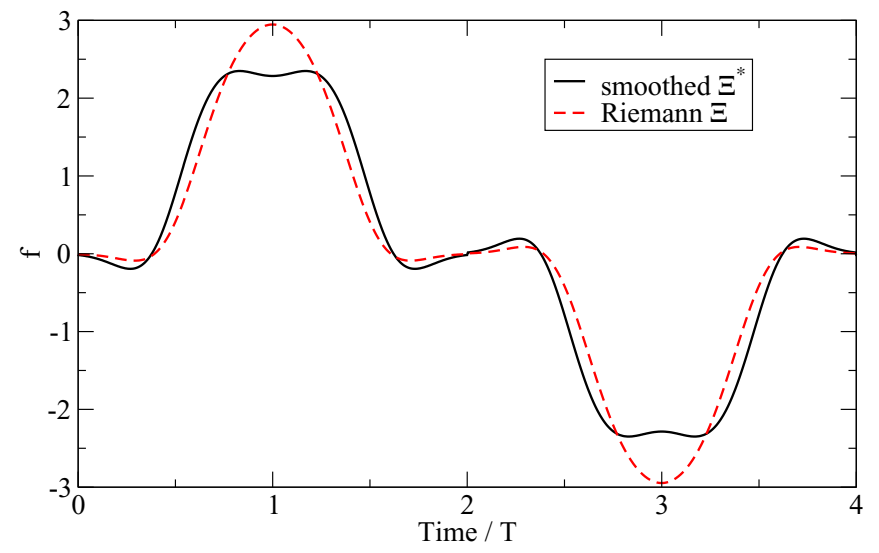

FIG. 5. (Color online) Although the driving functions $f(t)$ that produce $\Xi^{*}(E)$ and $\Xi(E)$ look superficially very similar, they differ in details. We show here the driving functions for $E=4$ for the two Riemann functions we consider.

location of zeros for $\Xi(E)$ and $\Xi^{*}(E)$, demonstrating the wellknown analytical sensitivity of the zeta function.

\section{RESULTS}

\section{A. High-frequency limit}

The expression for the effective tunneling, $J_{\text {eff }}$, that we use in this work comes from a first-order perturbation theory calculation of the driven system. In this perturbation theory, the "small parameter" is $J / \omega$, and so this result is only valid in the high-frequency limit $\omega \gg J$. This would require the driving period $T=2 \pi / \omega$ to be small; however, this contradicts the requirement that $T$ be as large as possible so that the integrals (8) and (12) are well converged. To be able to satisfy both these requirement, we therefore scale the driving as $f(t) \rightarrow \Omega f(\Omega t)$, where $\Omega>1$, while keeping $T_{0}=2 \pi$ constant. Note that this is not a trivial rescaling of time, because its effect is to make the pulses shown in Fig. 2(b) narrower and taller, while keeping their spacing constant at $T$.

We show the effect of increasing $\Omega$ on the pulse shape in Fig. 6(a). It can be clearly seen that the pulses become progressively more localized and of higher amplitude. As the pulses become shorter and more intense, the quasienergy spectrum of the system approaches that of the high-frequency limit, as we show in Fig. 6(b). In particular we can see that the locations of the quasienergy crossings converge toward the zeros of the Riemann function. This evolution of the quasienergy spectrum as the driving frequency increases is a general feature of periodically driven systems including, for example, sinusoidal, square-wave, and triangular driving [14].

In principle $\Omega$ should be made as large as possible to ensure that the system is well within the high-frequency regime. We can see from Fig. 6(b) that good precision is obtained for $\Omega>4$, and the results we present below use $\Omega=128$. Increasing $\Omega$ beyond this value was found to introduce instabilities in the numerical integration of the system's time evolution.
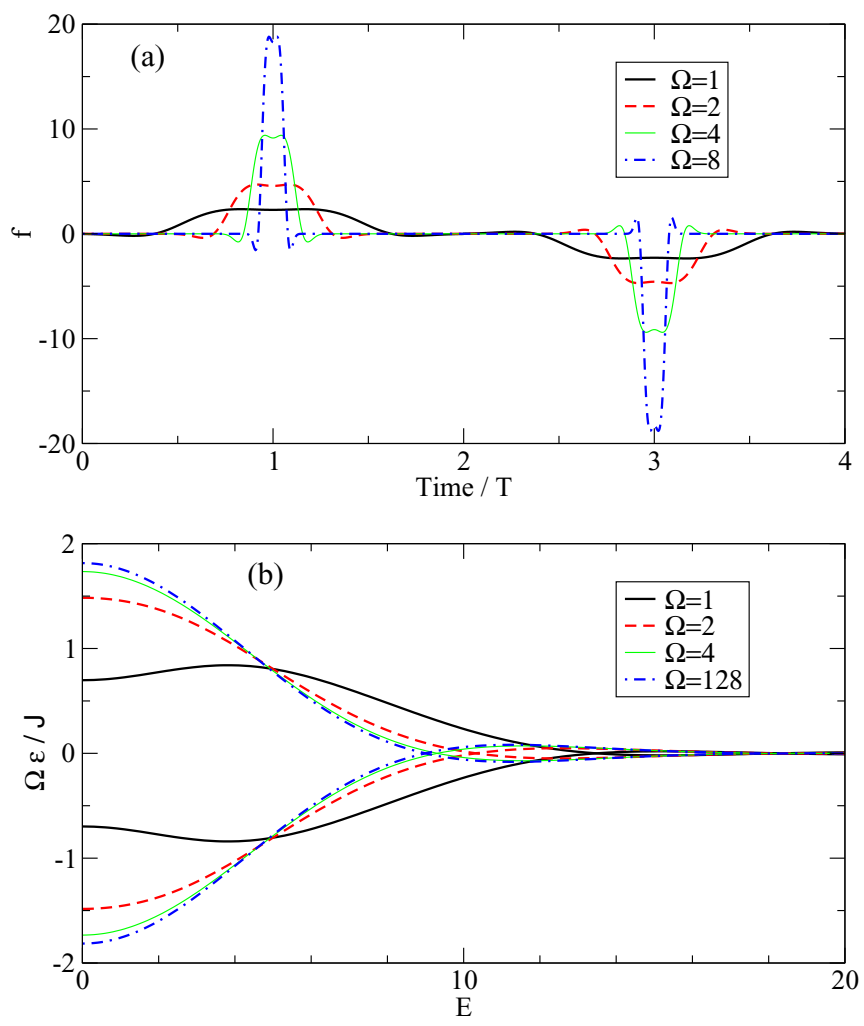

FIG. 6. (Color online) (a) Change of the driving function as $\Omega$ is increased. The pulses become progressively narrower and taller, while their spacing remains the same. (b) As $\Omega$ increases, the quasienergy spectrum asymptotically approaches that of the infinite-frequency limit, and the quasienergy crossings move towards the zeros of the Riemann function. Here we show the quasienergies for a system driven to reproduce the smoothed Riemann function, $\Xi^{*}(E)$. Note how the first crossing asymptotically approaches the first zero of $\Xi^{*}(E)$ at $E=8.993$ as $\Omega$ increases.

\section{B. Quasienergies}

In Fig. 7(a) we compare the quasienergies, obtained by the direct integration of the equation of motion (1) under the driving potential given in Eq. (10), with the exact behavior of $\Xi^{*}(E)$. The agreement is seen to be excellent. Similarly in Fig. 7(b) we compare the Riemann $\Xi$ function (11) with the quasienergies resulting from driving the system with the potential given by Eq. (14), and again see essentially perfect agreement. As the $\Xi$ functions decay roughly exponentially with $E$ [21], we show in Fig. 7(c) the same data plotted logarithmically. The cusps visible in this plot correspond to zeros of the $\Xi$ functions and thus to crossings of the quasienergies at which $J_{\text {eff }}$ vanishes. We see that the quasienergies accurately reproduce the behavior of the $\Xi$ functions over at least six orders of magnitude, although eventually precision effects do lead to deviations at large values of $E$.

\section{Measuring the effective tunneling}

A way of directly measuring $J_{\text {eff }}$ in experiment is to observe the expansion of a gas of cold atoms [16,18,24]. If the atoms are held in an optical lattice potential they can be described 


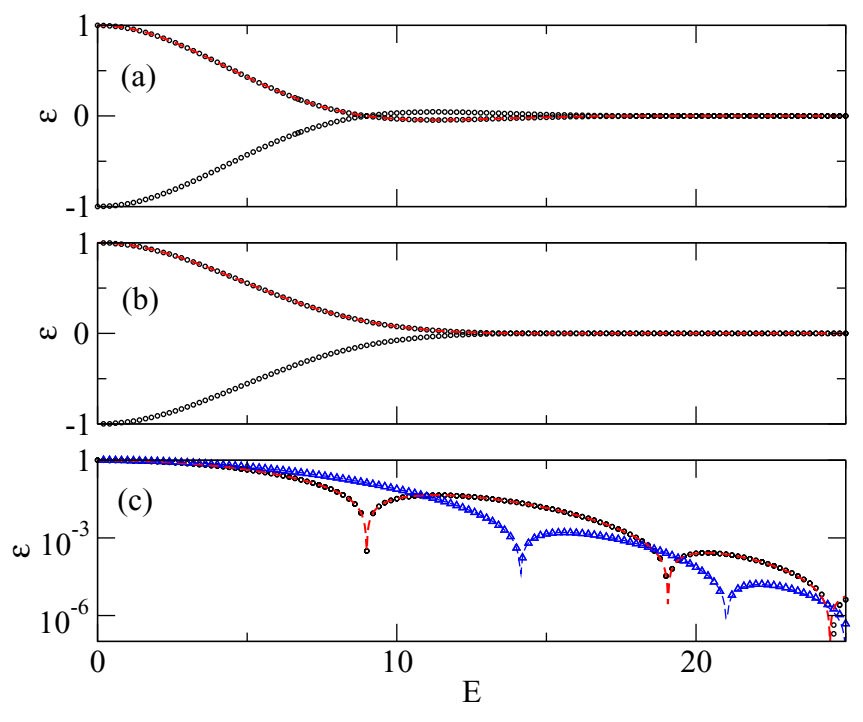

FIG. 7. (Color online) (a) Black symbols show the quasienergies of the two-level system driven by the potential $f(t)$ given by Eq. (10), the dashed (red) line is the smoothed Riemann function $\Xi^{*}(E)$. The quasienergies are normalized with respect to the quasienergies at $E=0$. The agreement between the two is excellent. (b) As above, but for the true Riemann $\Xi$ function, with the driving potential given by Eq. (14). Again, the results agree perfectly. (c) Comparison on a logarithmic scale. The first three zeros of $\Xi^{*}(E)$, corresponding to degeneracies of the quasienergies, are clearly visible as the downward-pointing cusps [black circles, red (thick) dashed line], as are the first two zeros of $\Xi(E)$ [blue triangles, blue (thin) dashed line] at $E \simeq 14.1347$ and 21.0220. The quasienergies reproduce the behavior of the $\Xi$ functions to a high degree of accuracy.

well by a tight-binding model

$$
H_{0}=-J \sum_{\langle i, j\rangle}\left(a_{i}^{\dagger} a_{j}+\text { H.c. }\right)+\sum_{j} V\left(r_{j}\right) n_{j},
$$

where $i$ labels the lattice site, $\langle i, j\rangle$ are nearest neighbors, and $V(r)$ is a trap potential. By "shaking" the optical lattice [25] it is possible to introduce a time-periodic driving potential $H(t)=H_{0}+f(t) \sum_{j} r_{j} n_{j}$ which generalizes Eq. (1) from a two-level model describing two sites to the case of $N$ lattice sites. For a parabolic trap potential, the initial state of the system will be Gaussian. If the trap potential is then released, this Gaussian wave packet will undergo free expansion at a rate governed by $\left|J_{\text {eff }}\right|[24]$.

In Fig. 8(a) we show the spread of the wave packet with time, $\sigma(t)=\left(\left\langle x^{2}\right\rangle-\langle x\rangle^{2}\right)^{1 / 2}$, under the periodic driving corresponding to $\Xi^{*}(E)$. For $E=0$ the wave packet expands rapidly and soon enters the ballistic regime in which $\sigma \propto t$. For $E=4$ the expansion is slower since $J_{\text {eff }}$ is smaller, and for $E=9$ the wave packet barely expands at all, indicating that this value of $E$ is close to a zero of $\Xi^{*}(E)$. In principle one would expect only partial destruction of tunneling, even when $E$ is tuned exactly to a zero of $\Xi^{*}$, due to the presence of longerranged hopping elements. Such an effect is seen in sinusoidally driven systems [18] where the band strongly narrows but does not collapse totally. For the case of the drivings we consider, the longer-ranged hoppings are suppressed considerably more
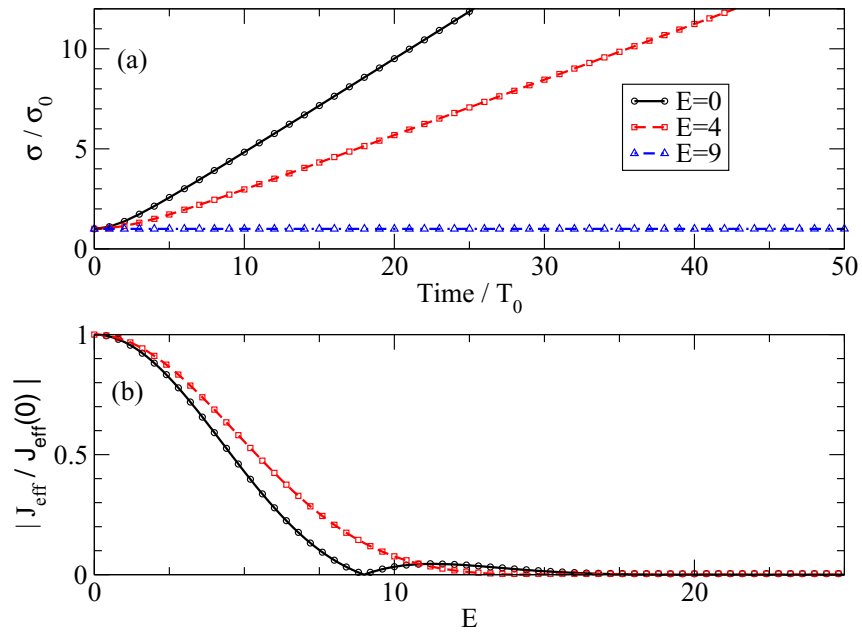

FIG. 8. (Color online) (a) Expansion of a Gaussian wave packet in an optical lattice with $N=200$ sites under the periodic driving potential $f(t)(10)$, such that $J_{\text {eff }} \propto \Xi^{*}(E)$. $T_{0}$ is the total driving period; $T_{0}=4 T$. For $E=0$ the wave packet spreads quickly, but for $E=4$ the wave packet spreads less rapidly, indicating that $J_{\text {eff }}$ is smaller. For $E=9$ the wave packet hardly expands at all, indicating that CDT is occurring. (b) The solid black curve shows $\left|\Xi^{*}(E)\right|$, and the black circles show the values of $\left|J_{\text {eff }}\right|$ measured from the expansion curves. The agreement between the two is excellent. Similarly the dotted red line shows $|\Xi(E)|$, and the red squares show the measured values of $\left|J_{\text {eff }}\right|$ for a system driven by the potential (14). Again the agreement is perfect.

than for the sinusoidal case; however, meaning their effect is essentially negligible.

These estimations can be made quantitative. In Fig. 8(b) we show the values of $\left|J_{\text {eff }}\right|$ obtained by measuring the spread of the wave packet after an expansion time of $50 T_{0}$ and fitting it to the result $\left|J_{\text {eff }}\right| \propto\left[\sigma(t)^{2}-\sigma_{0}^{2}\right]^{1 / 2}$, where $\sigma_{0}$ is the initial width of the condensate. Both of the driving potentials show excellent agreement with the exact $\Xi$ functions, demonstrating that measuring the expansion of a trapped cold atom system is a viable method to experimentally determine the zeros of the Riemann functions.

\section{CONCLUSIONS}

We propose an approach for a physical realization of the Riemann zeros. The main idea is to use a time-dependent driving potential to modify the dynamics of a quantum system, so that its quasienergy spectrum mimics the desired Riemann function. We provide a systematic scheme for calculating the appropriate potential and suggest a physical realization using driven cold atoms. Although the observation of high Riemann zeros is hindered by the rapid decay of the $\Xi$ function, this could be compensated in experiment by increasing the tunneling strength (which depends exponentially on the optical lattice depth), or by using a form of the zeta function which decays more slowly, such as $\xi(s) / s[7,8]$. An intriguing possibility for future study would be to apply this technique to more general trigonometric integrals of the function $\Phi(t)$, which could thereby lead to the measurement of a new bound on the de Bruijn-Newman constant, the value of which is related to the Riemann hypothesis [26]. 


\section{ACKNOWLEDGMENTS}

The authors thank Michael Berry for stimulating discussions. C.E.C. was supported by the Spanish MINECO through
Grants No. FIS2010-21372 and No. FIS2013-41716-P, and G.S. by Grant No. FIS2012-33642, QUITEMAD, and the Severo Ochoa Programme under Grant SEV-2012-0249.
[1] G. Sierra, A Physics Pathway to the Riemann Hypothesis, in Mathematical Physics and Field theory. Julio Abad, in Memoriam., edited by M. Asorey, J. V. Garcia-Esteve, M. F. Rañada, and J. Sesma (University of Zaragoza Press, Zaragoza, 2009), pp. 383-390; G. Sierra, arXiv:1012.4264.

[2] D. Schumayer and D. A. W. Hutchinson, Rev. Mod. Phys. 83, 307 (2011).

[3] H. L. Montgomery, Proc. Symp. Pure Math. 24, 181 (1973).

[4] A. M. Odlyzko, Math. Comput. 48, 273 (1987).

[5] M. V. Berry, in Quantum Chaos and Statistical Nuclear Physics, edited by T. H. Seligman and H. Nishioka, Springer Lecture Notes in Physics Vol. 263, p. 1 (Springer, New York, 1986).

[6] J. P. Keating, in Supersymmetry and Trace Formulae: Chaos and Disorder, edited by I. V. Lerner et al. (Kluwer Academic/Plenum Publishers, New York, 1999), pp. 1-5.

[7] B. van der Pol, Bull. Am. Math. Soc. 53, 976 (1947).

[8] M. V. Berry, J. Phys. A: Math. Theor. 45, 302001 (2012).

[9] C. Feiler and W. P. Schleich, New J. Phys. 15, 063009 (2013).

[10] G. Pólya, Acta Math. 48, 305 (1926).

[11] H. M. Edwards, Riemann's Zeta Function (Academic Press, New York, 1974).

[12] F. Grossmann, T. Dittrich, P. Jung, and P. Hänggi, Phys. Rev. Lett. 67, 516 (1991).

[13] M. Grifoni and P. Hänggi, Phys. Rep. 304, 229 (1998).

[14] C. E. Creffield, Phys. Rev. B 67, 165301 (2003).

[15] J. H. Shirley, Phys. Rev. 138, B979 (1965).
[16] H. Lignier, C. Sias, D. Ciampini, Y. Singh, A. Zenesini, O. Morsch, and E. Arimondo, Phys. Rev. Lett. 99, 220403 (2007).

[17] E. Kierig, U. Schnorrberger, A. Schietinger, J. Tomkovic, and M. K. Oberthaler, Phys. Rev. Lett. 100, 190405 (2008).

[18] A. Eckardt, M. Holthaus, H. Lignier, A. Zenesini, D. Ciampini, O. Morsch, and E. Arimondo, Phys. Rev. A 79, 013611 (2009).

[19] Equation (4) contains a extra factor of four as compared with a similar expression appearing in Ref. [20] in order to agree with the original expression used by Pólya in Ref. [10].

[20] E. C. Titchmarsh, The Theory of the Riemann Zeta Function (Oxford University Press, Oxford, 1986).

[21] G. Sierra and J. Rodríguez-Laguna, Phys. Rev. Lett. 106, 200201 (2011).

[22] G. Sierra, J. Phys. A: Math. Theor. 47, 325204 (2014).

[23] Since we employ the relation $F(t)=\cos ^{-1} \phi(t)$, we clearly require $|\phi(t)| \leqslant 1$ for all $t$. It can be shown that a necessary and sufficient condition for this is $a^{2}<x$, where $x$ and $a$ parametrize the modified Bessel function (4). For the case we are interested in, $a=9 / 4$ and $x=2 \pi$ (e.g., $2.25<2.50$ ), the relation $a<\sqrt{x}$ is indeed satisfied.

[24] C. E. Creffield, F. Sols, D. Ciampini, O. Morsch, and E. Arimondo, Phys. Rev. A 82, 035601 (2010).

[25] N. Goldman and J. Dalibard, Phys. Rev. X 4, 031027 (2014).

[26] A. M. Odlyzko, Numer. Algorithms 25, 293 (2000). 